\title{
Quasi Fermi Level Scan of Band Gap Energy in Photojunction
}

\author{
B.A. Orlowski ${ }^{a} *$, K. Gwóźdź ${ }^{b}$, M. GalickA $^{a}$, S. Chusnutdinow ${ }^{a}$, E. Placzek-Popko ${ }^{b}$, \\ M.A. Pietrzyk ${ }^{a}$, E. Guziewicz ${ }^{a}$ And B.J. KowAlski ${ }^{a}$ \\ ${ }^{a}$ Institute of Physics, Polish Academy of Sciences, Aleja Lotnikow 32/46, PL-02668 Warsaw, Poland \\ ${ }^{b}$ Department of Quantum Technology, Faculty of Fundamental Problems of Technology, \\ Wroclaw University of Science and Technology, Wybrzeze Wyspianskiego 27, 50-370 Wroclaw, Poland
}

(Received April 9, 2018; in final form June 28, 2018)

\begin{abstract}
Photovoltage generation model results are compared with the correlated illumination intensity spectra of semiconductors photojunction. The moderate continuous increase of illumination intensity of semiconductor photojunction leads to remarkable increase of relative concentration of minority carriers and related to it quasi Fermi level scan along the energy band gap. The scanning energy region runs up from thermal equilibrium Fermi level for electrons and down for holes. For moderate illumination related changes of quasi Fermi levels energy of minority carriers dominate over the changes of majority carriers and they decide on measured open circuit voltage. Expected spectrum of quasi Fermi level scan on illumination intensity will strongly depend on interaction with electronic "defects" located in photojunction region (e.g. impurities, clusters, barriers, etc.) leading to the majority quasi Fermi level pinning. Measured region of quasi Fermi level energy pinning allows to estimate the defect states parameters (binding energy and concentration) in situ during the work of photojunction. The theoretical model of described effect will be presented and supported by experimental data measured for $\mathrm{Si} p / n$ junction and $\mathrm{CdTe} /$ ZnTe heterojunction.
\end{abstract}

DOI: 10.12693/APhysPolA.134.590

PACS/topics: 73.40.Lq

\section{Introduction}

Continuously ongoing advanced studies aimed at indepth understanding of the physics of photovoltaic heterojunctions [1-9] and improvement of construction of solar cells $[10-15]$ and ultraviolet sensitive photodetectors $[16-18]$ are in line with the search of renewable energy sources supporting the sustainable civilization development. The comprehensive theory of solar cells exists and can be found in textbooks [3-6] convenient models describing particular aspects of electricity generation in a solar cell, facilitating experimental data interpretation or useful as an enlightening example are still welcome.

In this paper we propose a model describing effect of photovoltage generation by the analysis of the quasi Fermi level shifts induced by properly selected parameters of illumination (lasers beam with properly selected $h \nu$ and intensity). It is also demonstrated that the model can be used for interpretation of the data derived from the open circuit voltage measurements. Limitations related to the presence of electrically active defects in the cell can easily be revealed by comparison of the data with the results of model calculations.

Photovoltaic heterojunctions are built of two kinds of semiconductor materials with two kinds of electrons and holes electronic structure in the conductions and valence bands on each side of the junction. In general, we have a junction of two semiconductors with different crystalline

*corresponding author; e-mail: orbro@ifpan.edu.pl structures, different electronic band structures, and different minority and majority carriers at either side. Let us assume that under illumination the same density of $n$ and $p$ carriers is generated at each illuminated point of heterojunction. Even so, this leads to a remarkably higher relative increase of concentration for minority than for majority carriers and consequently to a different changes of the corresponding quasi Fermi levels energies. Under illumination, the differences of the change of quasi Fermi energies for the same type of carriers (electrons or holes) contributes to create total photovoltage.

The first part of the paper discusses photovoltage generation in terms of basic correlations between the main fundamental parameters, such as: the changes of minority and majority carriers concentration, and the related changes of quasi Fermi energies of minority and majority carriers. The second part illustrates the correspondence between the predicted open circuit photovoltage spectra and the results of corresponding experiments, with deviations caused by defects in the semiconductors junction region.

\section{The model presentation}

The heterojunction of two semiconductors with different band gaps, band offsets and type of carriers were analyzed in many works, like those of Fan [1], Anderson [3] or Sze [4]. Figure 1 presents a simplified picture of the changes of quasi Fermi levels of minority and majority carriers relatively to the thermal equilibrium Fermi level energy, just to concentrate attention on changes of these parameters under illumination. The region of the junction is depicted by a broken line, and can be deformed 
by particular material parameters and defects occurring during the growth processes. It will allow to study the influence of particular e.g. nano defects introduced to the junction region and compare it with spectra predicted by the model for the parameters of these particular defects.

In the $n$-type semiconductor (side 1 ) the thermal equilibrium Fermi level is located far from the valence band edge, whereas in the $p$-type semiconductor (side 2) the $F$ level is located far from the conduction band edge. Under illumination, the scan of quasi Fermi level of minority carriers can occur in the wide energy range of related band gaps.

Let us consider the case when the incident light of $E_{g 2}<h \nu<E_{g 1}$ is absorbed only at the side 2 of the junction. Illumination of part 2 destroys the thermal equilibrium condition of carriers and changes it to the steady state condition. The equal numbers of electrons and holes generated in part 2 increase the minority electron concentration in the conduction band from $n_{20}$ to $n_{21}$ and the majority holes concentration in the valence band from $p_{20}$ to $p_{21}$. To describe the electron concentration in part 2 a quasi Fermi level energy for electrons has to be introduced. This quasi Fermi level energy is shifted upwards relative to $F$ by a value of $F_{2 n}$ (see Fig. 1, side 2), adequate to the change of electron concentration. Similarly, for the holes generated in the valence band of side 2 a quasi Fermi level energy has to be introduced to shift down $F$ by $F_{2 p}$ (see Fig. 1, side 2).

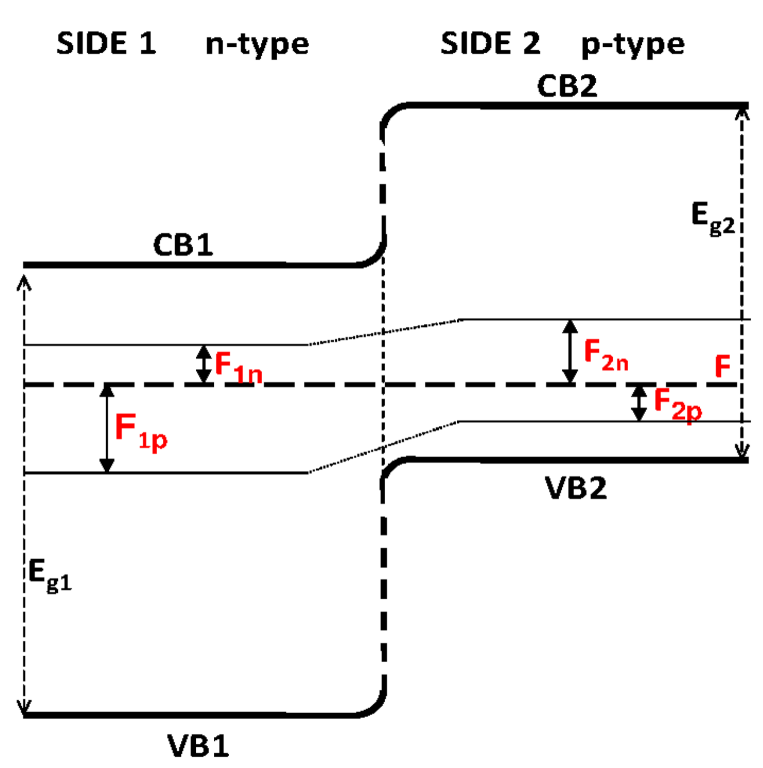

Fig. 1. Illustration of quasi Fermi levels changes in a schematic structure of a photovoltaic heterojunction. $F$ is the thermal equilibrium Fermi energy, $F_{1 p}$ and $F_{1 n}$ are relative changes of quasi Fermi levels of minority holes $p$ and majority electrons $n$ after generation of $n=p$ carriers on side 1 of the junction ( $n$-type, wide band gap $\left.E_{g 1}\right)$, whereas $F_{2 n}, F_{2 p}$ are the changes of quasi Fermi levels of minority electrons $n$ and majority holes $p$ on side 2 ( $p$-type, medium band gap $E_{g 2}$ ) [8].
By analogy, the shifts of quasi Fermi levels of electrons and holes in side 1 (under illumination with $h \nu>E_{g 1}$ ) are $F_{1 n}$ and $F_{1 p}$, respectively, for electrons and holes (see Fig. 1, side 1). Each relative change of the minority or majority carriers concentration is described by the corresponding shifts of their quasi Fermi levels. In consequence, under proper illumination of both sides of the heterojunction, there will be four values of steady state quasi Fermi energies: $F_{1 n}, F_{1 p}, F_{2 n}$, and $F_{2 p}$.

The difference of the electric potential on the two sides of the heterojunction for the electrons in conduction bands can be expressed by the difference of electrons quasi Fermi level shifts

$$
V_{n}=\left(F_{2 n}-F_{1 n}\right) / e,
$$

where $e$ is the electron charge.

For holes in the valence bands the electric potential difference will amount to

$$
V_{p}=\left(F_{1 p}-F_{2 p}\right) / e .
$$

The sum of the voltage $V_{n}$ and $V_{p}$ contributes to the total potential difference $V_{n p}$ with the same polarity and with the same direction of the electric field. The direction of the electric field (from side 1 to side 2 ) is determined by chemical potential differences in accordance with the Fermi-Dirac function: positive for side 1 and negative for side 2 . The free electrons and holes will be separated and the measured photo cell voltage will be minus on side 1 and plus on side 2 .

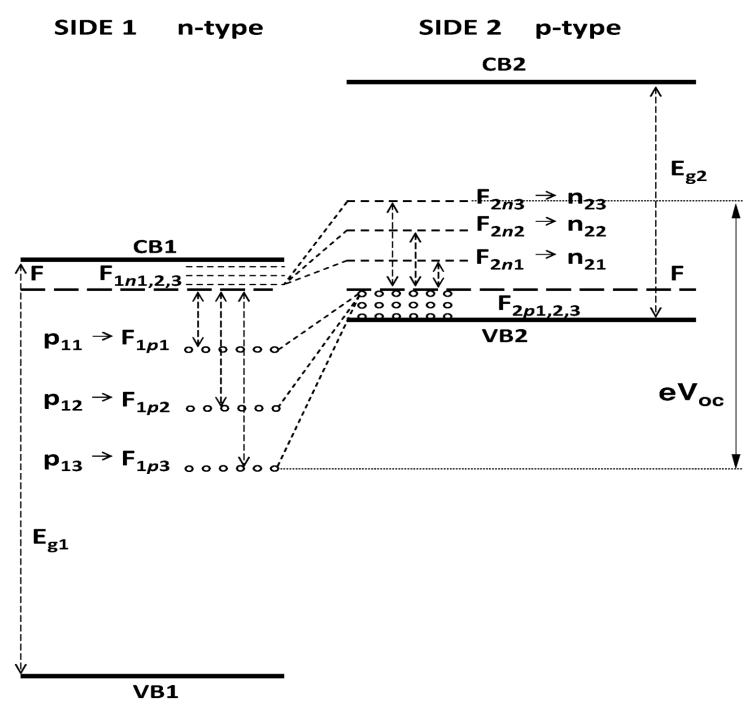

Fig. 2. The changes of quasi Fermi level positions corresponding to the sequential increase of generated carriers. For side 1 sequential increase of holes concentration from $p_{10}$ to $p_{11}, p_{12}$ and $p_{13}$ and electrons concentration from $n_{10}$ to $n_{11}, n_{12}$ and $n_{13}$ leads to the change of quasi Fermi levels energies of holes (minority carriers) to $F_{1 p 1}$, $F_{1 p 2}$ and $F_{1 p 3}$, and of electrons (majority) to $F_{1 n 1,2,3}$. Analogical changes will appear for side 2 with minority electrons and majority holes. The $V_{o c}$ in Fig. 2 neglects majority carriers contribution. 


\section{Change of carriers concentration and of quasi Fermi levels}

As already mentioned, in the photovoltaic effect the change of carrier concentration leads to a corresponding change of the quasi Fermi level and, consequently, to a change of the created open circuit photovoltage. The changes are described by the Fermi-Dirac function or by the Maxwell-Boltzmann function - as an approximation of the former.

Let us take the Fermi-Dirac function to determine the electron concentration $n_{20}$ in the conduction band for the density of states $N_{2 c}$, when the conduction band edge is located at a distance of $E_{2 c}$ from the Fermi level position

$$
n_{20}=N_{2 c} /\left\{\exp \left(E_{2 c} / k T\right)+1\right\},
$$

where $T$ is the temperature and $k$ - the Boltzmann constant.

In the case of $\exp \left(E_{2 c} / k T\right)$ dominating over 1 (for $T=293 \mathrm{~K}, k T=25.03 \mathrm{meV}$ ) the formula takes the form of the Maxwell-Boltzmann distribution function and the concentration can be expressed as

$$
n_{20}=N_{2 c} \exp \left(-E_{2 c} / k T\right) .
$$

Under illumination the concentration of minority electrons increases to the value $n_{21}$ and it is causing a shift of the quasi Fermi level $F_{2 n}$ towards the conduction band edge

$$
n_{21}=N_{2 c} \exp \left(\left(-E_{2 c}+F_{2 n 1}\right) / k T\right) .
$$

Dividing formula $(2.2)$ by $(2.1)$ we can correlate the change of minority electron concentration from $n_{20}$ to $n_{21}$ with the change of the quasi Fermi level $F_{2 n}$ [7]:

$$
F_{2 n}=k T\left(\ln \left(n_{21} / n_{20}\right)\right) \text {. }
$$

An analogical formula can be used for minority holes in side 1 of Fig. 1. The increase of hole concentration from the value $p_{10}$ to $p_{11}$ shifts the quasi Fermi level by $F_{1 p}$ towards the valence band edge

$$
F_{1 p}=k T\left(\ln \left(p_{11} / p_{10}\right)\right) \text {. }
$$

The same changes of the absolute value of the quasi Fermi energies will occur also for majority carriers.

Let us consider for how big change of carriers concentration a considerable change of the quasi Fermi level can be obtained. Assuming the increase of minority carriers concentration, e.g., electrons from $n_{20}=10^{6} \mathrm{~cm}^{-3}$ to $n_{21}=10^{7} \mathrm{~cm}^{-3}$ and $k T=26 \mathrm{meV}$ we obtain $F_{2 n}=$ $k T\left(\ln \left(n_{21} / n_{20}\right)\right)=59.88 \mathrm{meV}$ (approximately $60 \mathrm{meV}$ ). The same increase of majority carriers concentration (for photo generated carriers $n=p$ ) from $n_{10}=10^{17} \mathrm{~cm}^{-3}$ to $n_{21}=\left(10^{17}+10^{7}\right) \mathrm{cm}^{-3}$ at the same temperature leads to $F_{2 n}=k T\left(\ln \left(n_{21} / n_{20}\right)\right)$ which can be neglected as $n_{21} / n_{20}$ can be approximated by one. In this case the electric potential difference $V_{n}=\left(F_{2 n}-F_{1 n}\right) / e=F_{2 n} / e$ is related only to the change of the quasi Fermi level of minority electrons. Each subsequent relative increase of electrons concentration by a factor of ten $\left(n_{22} / n_{21}=\right.$ $n_{23} / n_{22}=10$ ) changes the quasi Fermi energy by $60 \mathrm{meV}$. The change of the minority carriers quasi Fermi level with increasing carriers concentration is illustrated in Fig. 2.
The dependence of the quasi Fermi level energy on minority carriers concentration is presented in Fig. 3. The quasi Fermi level scan along forbidden gap leads to the change of open circuit voltage generation (Figs. 2 and 3 ). The defect centers $N_{1 d}$ and $N_{2 d}$ located at the band gap scan region can act as recombination centers and reduce the concentration of generated minority carriers $p_{1}$ and $n_{2}$ on side 1 and side 2 , respectively. This will reduce the quasi Fermi energies $F_{1 p}$ and $F_{2 n}$ and will lower value of obtained open circuit voltage (see Fig. 4).

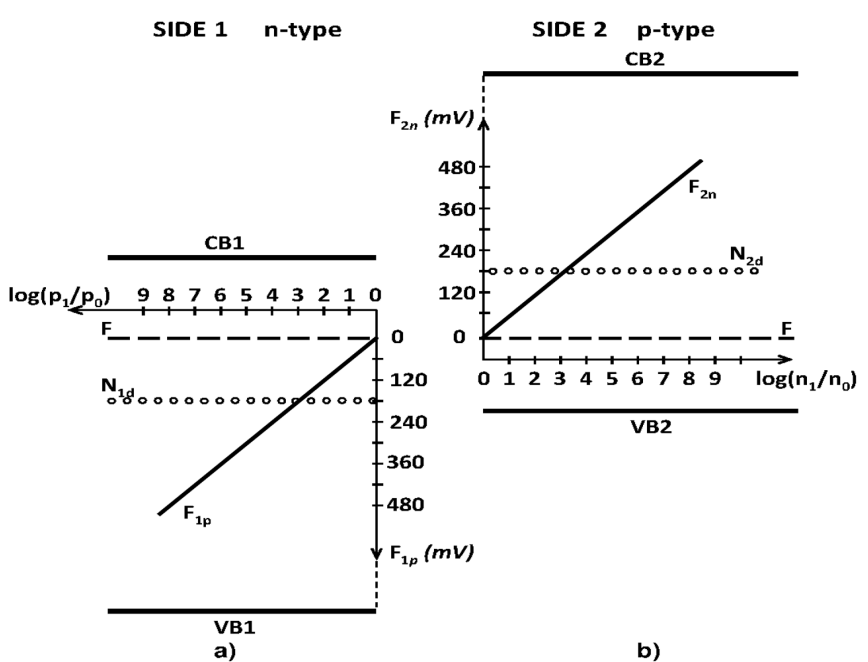

Fig. 3. (a) Holes quasi Fermi level change $F_{1 p}$ versus increase of minority holes concentration. (b) Electrons quasi Fermi level change $F_{2 n}$ versus increase of minority electron concentration. Open circles denote defect level states with concentrations of $N_{1 d}$ and $N_{2 d}$.

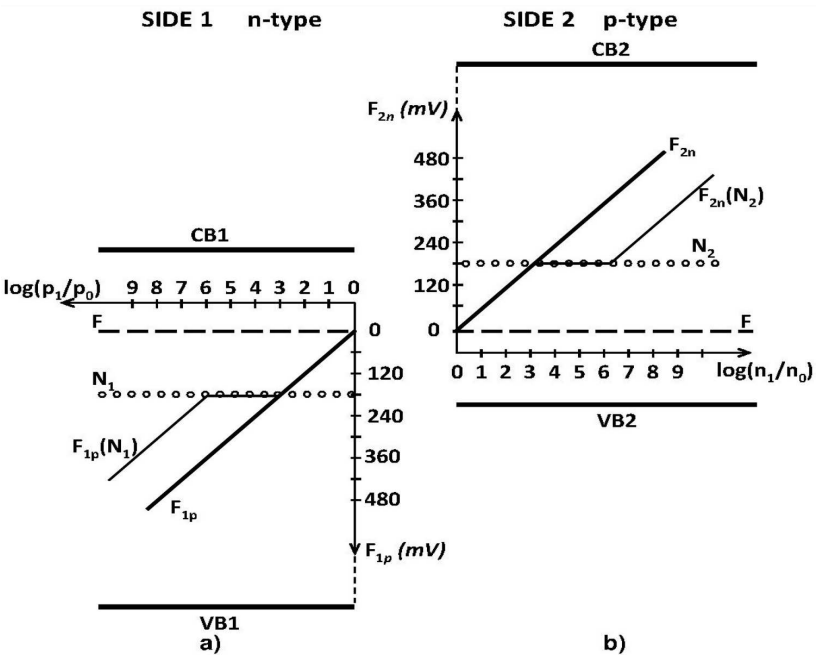

Fig. 4. Quasi Fermi level pinning in the presence of defect levels: (a) $N_{1}$ located at $180 \mathrm{meV}$ below the Fermi energy and (b) $N_{2}$ located $180 \mathrm{meV}$ above the Fermi energy. Thick line corresponds to the case without any defects. 
Let us consider the case when quasi Fermi level crosses the defect energy level and the pinning of the quasi Fermi level occurs at the energy of defect level energy position.

In Fig. 4a the linear dependence of the hole quasi Fermi level $F_{1 p}$ on value of hole concentration is presented. The slope of this linear dependence can be changed (as illustrated by the flat step region in Fig. 4a, for $F_{1 p}=180 \mathrm{meV}$ ) when the quasi Fermi level position approaches the energy level position of a defect with concentration of $N_{1}$. In this energy region an increase of hole concentration in the range from $10^{3}$ to $10^{6} \mathrm{~cm}^{-3}$ does not result in an increase of the quasi Fermi energy $F_{1 p}$. The photogenerated holes concentration is reduced by the parasitic effect of quasi Fermi level pinning. This effect can be caused by crystal lattice nanodefect states or impurity states correlated with minority $p$-type carriers.

An analogical case occurs for electrons in the conduction band of the side 2 (see Fig. $4 \mathrm{~b}$ ). Such pinning can occur for the range of generated electron concentrations and may stabilize the quasi Fermi level $F_{2 n}\left(N_{2}\right)$ at $180 \mathrm{meV}$. This effect can be caused by the defect states correlated to the minority $n$-type carriers and it will stop after saturation. Precise study of open circuit voltage vs. illumination intensity spectra can give information on the parameters of defects causing this parasitic effect.

Let us assume that the introduction of defects with the densities $N_{2 n 1}, N_{2 n 2}$ and $N_{2 n 3}$ (see Fig. 5) leads to the reduction of the density $n_{2}$ to the value $n_{21}=0.8 n_{2}$, $n_{22}=0.5 n_{2}$ and $n_{23}=0.2 n_{2}$, respectively. The reduction of the densities leads to decrease of the quasi Fermi level of related carriers and to decrease of the open circuit voltage (see Fig. 5).

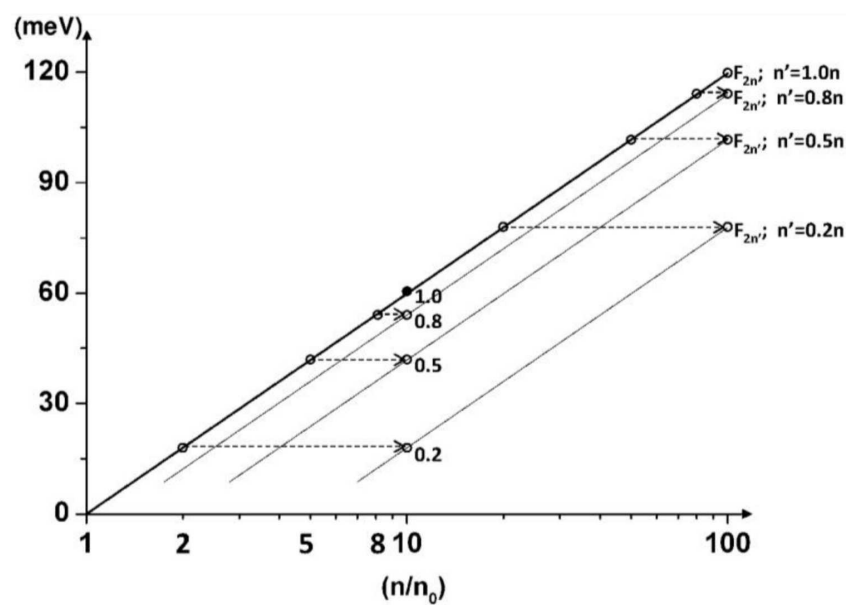

Fig. 5. Dependence of quasi Fermi level value $F_{2 n}$ on minority carrier concentration without any impurities (thick line) and in the presence of $N_{1 n 1}, N_{1 n 2}$, and $N_{1 n 3}$ impurities (thin lines).

Changing the parameters of the $N_{1 p}$ or $\mathrm{N}_{2 n}$ impurities, we can follow their influence on the reduction of the minority carrier concentration and correlate it with the reduction of the measured open circuit voltage.

\section{Experimental and model results comparison}

\subsection{Silicon $n / p$ junction open circuit voltage}

The silicon $n / p$ junctions were used to investigate experimental dependence of open circuit voltage value versus the number of illuminating photons. The epitaxial layer of Si $n$-type was grown on top of the Si $p$-type crystalline substrate. One of two studied identical samples possessed good crystalline orientation while the second one was properly etched to obtain pyramidal texture on the top surface.

As a source of monochromatic radiation of $\lambda=445 \mathrm{~nm}$ a laser with the power $P_{445}=53.5 \mathrm{~mW}$ was used. The number of photons illuminating the sample was changed by the exposition time of open shutter. The exposition time was changed in the range from $0.1667 \mathrm{~ms}$ up to $3.333 \mathrm{~ms}$ and it corresponds to the number of photons in the bunch illuminating the sample. During the time of open shutter the sample was illuminated and generated open circuit voltage value was measured. The experimental data obtained for the both Si $n / p$ samples (clean and etched) are presented in Fig. 6.

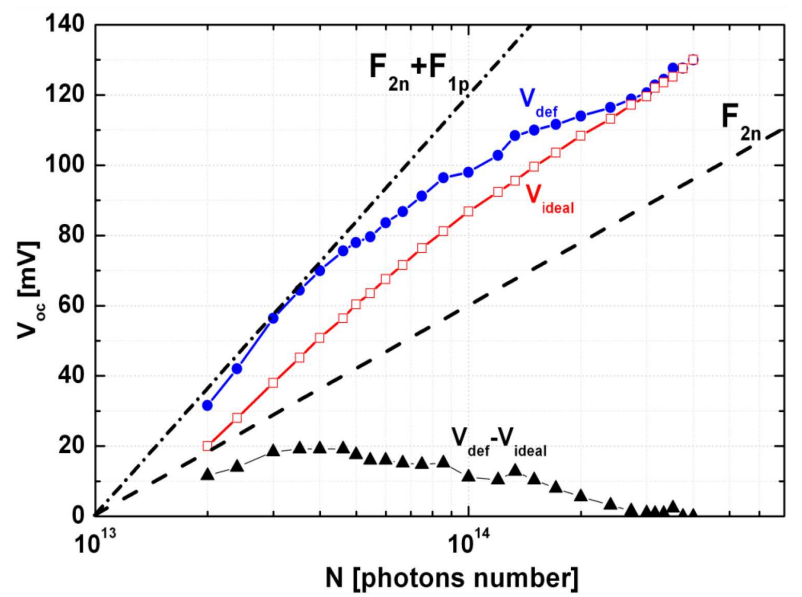

Fig. 6. Plot of measured $V_{o c}$ versus number $N$ of photons illuminating $\mathrm{Si} p / n$ junctions. The broken lines correspond to the model predicted dependence (lower one for single cell illumination $F_{1 n}$ and upper one for double $F_{1 n}+F_{2 p}$ cell illumination). The smooth continuous curve (rings) corresponds to the junction of good crystalline structure. The upper continuous curve (dots) corresponds to the identical sample but with properly etched $\operatorname{Si}(n / p)$ junction (destroyed crystalline surface possessing like pyramids elements structure). The lowest continuous curve (triangle) corresponds to the difference of the measured curves (etched minus clean).

The two broken lines in Fig. 6 correspond to the lines predicted by model in Fig. 3. The lower broken line corresponds to the case of only one cell illumination by absorbed radiation, when the absorbed radiation leads either to minority electrons or minority holes generation. The upper broken line corresponds to the case of radiation absorbed in both cells, and minority electrons and minority holes are generated. For the starting low number of photons illumination, the inclination 
of the measured both curves is surprisingly high. It can be expected that at this range of radiation intensity the light scattered at the surface contributes to the value of generated voltage and the both cells contributes to it. The scattering effect dominates the value of ideal generated voltage. At the next region of the radiation intensity the upper curve voltage values (for the structure with the pyramidal texture) continuously dominate over the values of lower curve (for the good crystalline structure). It can be expected that scattering of the light on the pyramidal texture will multiply the number of generated $p$ and $n$ minority carriers. Wide and smooth shape of the difference of the curves in the region of generated voltage between values 41 to $80 \mathrm{mV}$ well correspond to the effect of light scattering. The scattering again can dominate over small structures of both cells contribution to the photovoltage and any structure is difficult to be recognized at these scattering region. In the region of voltage from 80 to $97 \mathrm{mV}$ the upper curve is parallel to the lower curve.

At the two $V_{o} c$ regions 99 and $109 \mathrm{mV}$ the upper curve lowers inclination and from 109 to $120 \mathrm{mV}$ it approaches and follows the inclination of continuous lower curve. The last two effects can be treated as caused by interaction of defect states with the minority carriers during the crossing of quasi Fermi level with the level of defect states. The particular defects introduced to the region of heterojunction can lead to the creation of defects leading to the change of the quasi Fermi level position of minority carriers and it leads to the lowering of the open circuit photovoltage. These photovoltage pinning effect appears at the energy level located at $99 \mathrm{meV}$ over the thermal equilibrium Fermi level $F$ and corresponds to the region of photons number value from $8.8 \times 10^{13}$ to $1.05 \times 10^{13}$ of photons values. The next region of pinning starts from photovoltage $110 \mathrm{mV}$ and it corresponds to the change of $N$ value from $1.01 \times 10^{14}$ up to $1.2 \times 10^{14}$ of photons values. At the end the saturation of the defect states is expected, and upper curve comes to be parallel to the lower ideal curve.

In homojunction the band gap of both cells is equal and the number of illuminating photons is divided between upper and lower cell. In the case the both cells contribute to the inclination of measured curve $\left(F_{n}+F_{p}\right.$ of good crystalline junction) and it dominates inclination of lower broken line which corresponds to the one cell. It leads to the higher generated voltage in comparison to the case when the same number of photons will be absorbed by only one cell and only these one cell will generate the voltage. For illumination of heterojunction, mainly only one of the cells absorbs the photons (with the lower band gap) and gives the lower contribution to the generated photovoltage, e.g. $\mathrm{ZnO} / \mathrm{GaAs}, \mathrm{ZnO} / \mathrm{Si}$, that are GaAs or $\mathrm{Si}$, respectively.

\subsection{Photo-heterojunction $\mathrm{ZnTe} / \mathrm{CdTe}$}

The sample of ZnTe/CdTe heterojunction was obtained by the molecular beam epitaxy [16] from the sources of $\mathrm{Zn}, \mathrm{Cd}$ and Te.
The number of illuminating photons (Fig. 7) was determined by the steady intensity of the laser source and regulated by open time of chopper. The experimental curve $\left(V_{o} c\right)$ in Fig. 7 corresponds to the measured open circuit voltage dependence on the number of photons illuminating the sample. The broken curve dependence is predicted by model (Fig. 3). The low dotted line shows the difference between the measured and predicted by model curve. The peaks of the curve can correspond to the density of defects (e.g. nanoclusters or dangling bounds) of unknown origin, and distort continuous smooth shape of the broken curve. The junction was not chemically etched and the distortions of continuous line are smaller than it was for etched Si $n / p$ junction (Fig. 6), The energy positions corresponding to the expected defect levels (with respect to the Fermi level) are shown on the right hand side scale of $V_{o c}$.

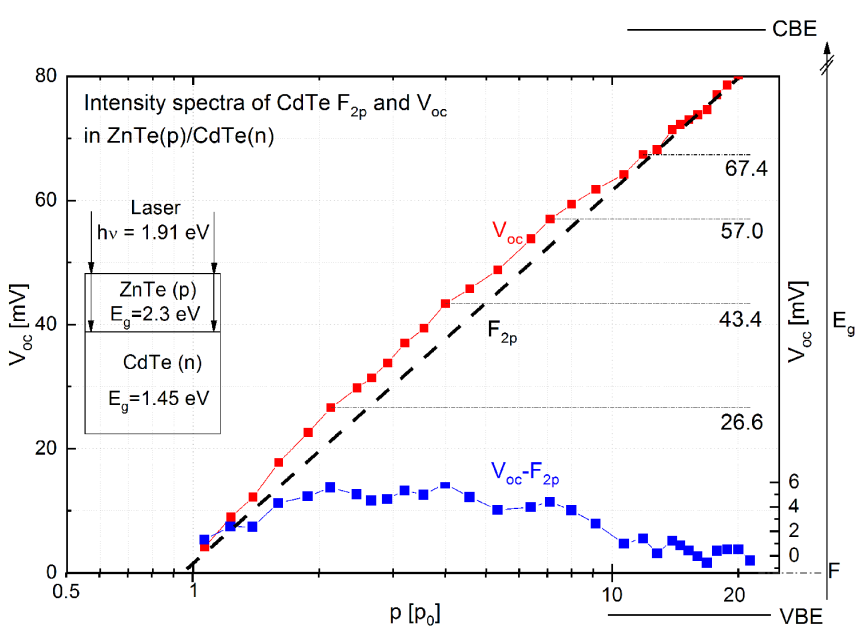

Fig. 7. Illumination intensity spectra of $\mathrm{ZnTe}(p) /$ $\operatorname{CdTe}(n)$ heterojunction expressed as a plot of $V_{o c}$ versus logarithm of generated concentration of minority carriers expressed in $n_{0}$ units ( $p_{0}$ - concentration of minority carriers in thermal equilibrium). The heterojunction is illuminated from the side of ZnTe, with photons of energy $h \nu=1.91 \mathrm{eV}$ sent from the laser source through the transparent ZnTe layer $\left(E_{g}=2.3 \mathrm{eV}\right)$ and absorbed by $\mathrm{CdTe}\left(E_{g}=1.45 \mathrm{eV}\right)$ crystal.

\section{Summary and conclusion}

The description of a photovoltaic junction model is based on the Fermi-Dirac function approximated by the Maxwell-Boltzmann equation. The predicted results of open circuit voltage dependence on illumination intensity are illustrated in Figs. 2 and 3. The modifications of predicted results by defect levels are presented in Fig. 4. The changes of minority and majority carrier concentrations leads to the scan of band gap by quasi Fermi level and it leads to the change of four related quasi Fermi levels of minority and majority carriers $\left(F_{1 n}, F_{2 n}, F_{1 p}\right.$, 
$\left.F_{2 p}\right)$. Total generated open circuit voltage is a sum of photovoltage generated in the electron cell $F_{2 n}-F_{1 n}$ and in the hole cell $F_{2 p}-F_{1 p}$. Proper choice of laser illumination can expose or exclude contribution of electron or hole cell to the total open circuit voltage. Under usually used illumination intensity the relative changes of minority carriers dominate over those of majority carriers. The difference of quasi Fermi energies in the conduction $\left(F_{2 n}-F_{1 n}\right)$ and valence $\left(F_{1 p}-F_{2 p}\right)$ bands, both contribute with the same polarity to the value of the total electric potential difference. The battery photovoltage polarity minus is created on the side 1 ( $n$-type), and polarity plus on the side 2 ( $p$-type).

The particular heterojunction growth technology parameters can lead to the introduction of particular defects to the junction region. These defects can be of crystalline structure origin electric barriers (e.g. mismatch of lattice parameters, precipitations, band offset, local structure, clusters), or impurities (e.g. traps, recombination centers). The value of generated open circuit voltage can be highly influenced by the defects which can reduce the concentration of generated minority carriers. The illumination intensity spectroscopy can help to distinguish the particular defects which reduce the concentration of generated minority carriers concentration and influence on the quality of the heterojunction photovoltage cells.

The illumination intensity spectra of photovoltage leads to the scan of quasi Fermi level along the semiconductors band gap and to the pinning of it by the "nanodefects" correlated to the nanostructures in the junction region. Presented theoretical model describe illumination intensity spectra and compares it with correlated experimental data. The method allows to determine parameters of the defects e.g. binding energy position relatively to the thermal equilibrium Fermi level and approximate value of its concentration.

\section{Acknowledgments}

This work was partly supported by the Polish National Science Centre (NCN) Grant No. UMO2016/21/B/ST5/03378 and by the Polish National Centre for Research and Development (NCBiR) through the project DZP/PBSII/1699/2013. Author K.G. would like to acknowledge the financial support in the form of the scholarship from the special-purpose grant awarded to Faculty of Fundamental Problems of Technology by Ministry of Science and Higher Education in 2016 for research and development of the young scientists and Ph.D. students.

\section{References}

[1] H.Y. Fan, Phys. Rev. 75, 1631 (1949).

[2] R.H. Bube, Photoconductivity of Solids, Wiley, New York 1960.

[3] R.L. Anderson, IBM J. 1960, 283 (1960).

[4] S.M. Sze, Semiconductor Devices: Physics and Technology, Wiley, 2001.

[5] S. Yang, Fundamentals of Semiconductors Devices, McGraw-Hill, New York 1978.

[6] J. Nelson, The Physics of Solar Cells, Imperial College Press, London 2003.

[7] L. Sosnowski, B. Orlowski, Phys. Status Solidi A 3, 117 (1970).

[8] B.A. Orlowski, A. Pieniazek, K. Goscinski, K. Kopalko, Acta Phys. Pol. A 129, 100 (2016).

[9] M.A. Pietrzyk, M. Stachowicz, D. Jarosz, R. Minikayev, M. Zielinski, P. Dluzewski, A. Kozanecki, J. Alloys Comp. 650, 256 (2015).

[10] M.A. Pietrzyk, E. Zielony, M.A. Stachowicz, A. Reszka, E. Placzek-Popko, A. Wierzbicka, E. Przezdziecka, A. Droba, A. Kozanecki, J. Alloys Comp. 587, 724 (2014).

[11] M.A. Pietrzyk, M. Stachowicz, A. Wierzbicka, A. Reszka, E. Przezdziecka, A. Kozanecki, Opt. Mater. 42, 406 (2015).

[12] R. Pietruszka, B.S. Witkowski, G. Luka, L. Wachnicki, S. Gieraltowska, K. Kopalko, M. Godlewski, E. Zielony, P. Bieganski, E. Placzek-Popko, Beilstein J. Nanotechnol. 5, 173 (2014).

[13] R. Pietruszka, B.S. Witkowski, S. Gieraltowska, P. Caban, L. Wachnicki, E. Zielony, K. Gwozdz, P. Bieganski, E. Placzek-Popko, M. Godlewski, Sol. Energy Mater. Sol. Cells 143, 99 (2015).

[14] E. Placzek-Popko, K.M. Paradowska, M.A. Pietrzyk, Z. Gumienny, P. Biegański, A. Kozanecki, J. Appl. Phys. 118, 074501-7 (2015).

[15] S. Chusnutdinow, R. Pietruszka, W. Zaleszczyk, V.P. Makhniy, M. Wiater, V. Kolkovsky, T. Wojtowicz, G. Karczewski, Acta Phys. Pol. A 126, 1072 (2014).

[16] E. Zielony, K. Olender, E. Placzek-Popko, T. Wosiński, A. Racino, Z. Gumienny, G. Karczewski, S. Chusnutdinow, J. Appl. Phys. $\mathbf{1 1 5}$ 244501 (2014).

[17] E. Przezdziecka, A. Wierzbicka, P. Dłuzewski, M. Stachowicz, R. Jakiela, K. Goscinski, M.A. Pietrzyk, K. Kopalko, A. Kozanecki, Phys. Status Solidi A 211, 2072 (2014).

[18] E. Przeździecka, S. Chusnutdinov, E. Guziewicz, D. Snigurenko, M. Stachowicz, K. Kopalko, A. Reszka, A. Kozanecki, J. Phys. D 48, 325105-1 (2015). 